\title{
On the variation of water diffusion coefficient in stratum corneum with water content
}

\author{
XIN LI ${ }^{a}$, ROBERT JOHNSON $^{\mathrm{b}}$, GERALD B. KASTING ${ }^{\mathrm{c}, *}$ \\ ${ }^{a}$ UC-P\&G Simulation Center, University of Cincinnati, Cincinnati, Ohio 45220 \\ ${ }^{\mathrm{b}}$ Research and Development Department., The Procter \& Gamble Company, Cincinnati, Ohio 45069 \\ ' James L. Winkle College of Pharmacy, University of Cincinnati, PO Box 670004, Cincinnati, Ohio 45267
}

${ }^{a}$ Current address: Process Systems Enterprise Inc. Cedar Knolls, NJ 07927

\section{"Correspondence:}

Gerald B. Kasting

James L. Winkle College of Pharmacy

University of Cincinnati Academic Health Center

P.O. Box 670004

Cincinnati, Ohio 45267-0004

Gerald.Kasting@uc.edu 


\section{ABSTRACT}

Water permeability and transient water sorption data in human and porcine stratum corneum (SC) are analyzed in conjunction with equilibrium water sorption data and a dynamic skin swelling model to develop a quantitative model for water diffusivity in the SC as a function of its water content. The recommended function (Model 1 ) is phenomenological and treats the SC as a uniform, swellable slab. This approach yields satisfactory agreement with experimental data over a wide range of $\mathrm{RH}$ and associated equilibrium SC water content, $C_{\mathrm{w}}$. It is supported by two alternative approaches. Model 2 considers the SC to be a multilaminate membrane consisting of alternating lipid and protein layers. Diffusivity in the protein phase is estimated from water diffusivity in other keratinized tissues, whereas diffusivity in the lipid phase is assumed to be linearly related to the swelling strain on intercellular lipids. Model 3 employs an analysis previously suggested by Stockdale to rationalize transepidermal water loss data in humans over a wide range of relative humidity. All models yield similar results for $0.20 \leq C_{\mathrm{w}} \leq$ $0.78 \mathrm{~g} / \mathrm{cm}^{3}$, the usual range of SC water content in vivo.

Keywords: biophysical models, hydration, mathematical model, skin, water sorption 


\section{INTRODUCTION}

Water diffusion in the skin's outermost layer, the stratum corneum (SC), has been studied for many years due to its importance in the conservation of body fluids and in skin health. ${ }^{1-12} \mathrm{~A}$ frequently studied special case is steady-state diffusion across fully hydrated skin, as summarized by Johnson et al. ${ }^{13}$ Related studies have examined the distribution of water in the skin under a variety of conditions. , $^{32,14-19}$ It is clear from this work that high levels of skin hydration are associated with a high water diffusion coefficient, as well as those of other permeants, ${ }^{20}$ in the SC. The origin of this concept may, in fact, be traced back much farther see Grice et al. ${ }^{21}$ and references therein, Scheuplein and Ross, ${ }^{22}$ Cooper and Van Duzee ${ }^{23}$ and Weil and Princen. ${ }^{24}$ A number of workers have attempted to quantify this dependence in the form of predictive relationships. ${ }^{2,4,5,8,12}$ In a similar vein, others have quantified the equilibrium water sorption capacity of SC in the form of adsorption isotherms., ${ }^{3,10,25,26}$ We recently developed a dynamic skin swelling model that incorporates both transient and equilibrium data on water sorption in the SC. ${ }^{27}$ In order to do this, an updated model for water diffusivity in the $\mathrm{SC}$ was required. This report describes the data selection and analysis procedure leading to the water diffusivity model employed in that report (Eq. 13 in Ref. 27). We furthermore support the phenomenological model with a mechanistic model involving alternating layers of lipids and corneocytes and a heuristic model based on the analysis of Stockdale. ${ }^{8}$

The phenomenological model assumes a homogeneous SC structure in which both water activity and diffusivity have the same dependence on water content throughout the tissue. This assumption can arguably be challenged - see, for example, the recent three-layer SC model presented by van Logtestjin et al. ${ }^{12}$ in which water handling properties vary with depth. Our 
analysis shows that it is not necessary to sub divide the SC into compartments to explain most features of water transport in the skin. A quantitative understanding of water transport and swelling in skin is furthermore a stepping stone towards better interpretation of hydration effects on the permeability of drugs and other compounds ${ }^{20}$ and the more complex swelling and permeability effects induced by binary solvent systems in which one of the components is water. $^{28-32}$

\section{THEORY}

\section{SC equilibrium water content}

The equilibrium water adsorption volume, $V$ (g water/g dry tissue), in the SC membrane was calculated according to the Guggenheim-Anderson-deBoer (GAB) isotherm, ${ }^{26}$

$$
\frac{V}{V_{\mathrm{m}}}=\frac{c k a_{\mathrm{w}}}{\left(1-k a_{\mathrm{w}}\right)\left(1-k a_{\mathrm{w}}+c k a_{\mathrm{w}}\right)}
$$

In Eq. 1, $a_{\mathrm{w}}$ is water activity, $c$ is a constant related to the difference in water binding enthalpy between the first and subsequent layers, $k$ is a constant with a value less than one that limits sorption at high values of $a_{\mathrm{w}}$, and $V_{\mathrm{m}}$ is the volume of an adsorbed monolayer. The associated

water concentration, $C_{\mathrm{w}}\left(\mathrm{g} / \mathrm{cm}^{3}\right)$, is related to $V$ according to Eq. (2), ${ }^{25}$

$$
C_{\mathrm{w}}=\rho_{\mathrm{w}} \frac{\rho_{\mathrm{mem}} V}{\rho_{\mathrm{w}}+\rho_{\mathrm{mem}} V}
$$

where $\rho_{\text {mem }}$ is the dry tissue density, $1.3 \mathrm{~g} / \mathrm{cm}^{325}$ and $\rho_{\mathrm{w}}$ is water density, $1.0 \mathrm{~g} / \mathrm{cm}^{3}$.

\section{Steady state water permeability and diffusivity}

Water permeability, $k_{\mathrm{p}}$, is defined as

$$
f_{\mathrm{ss}}=k_{\mathrm{p}} \Delta C_{\mathrm{ext}}
$$


where $f_{\mathrm{ss}}$ is steady state water flux in $\mathrm{g} / \mathrm{cm}^{2} \mathrm{~s}, \Delta C_{\text {ext }}$ is water concentration difference external to the $\mathrm{SC}$ membrane in $\mathrm{g} / \mathrm{cm}^{3}$. In a radiolabeled experiment with water or buffer on both sides of the tissue, water concentration is uniform in SC. So water diffusivity $(D)$ is constant throughout the $\mathrm{SC}$ and there is no convective flux. The steady state radiolabeled water flux $\left(f_{\mathrm{ss}}\right)$ is therefore the diffusive flux,

$$
f_{\mathrm{ss}}=D \frac{\Delta C_{\mathrm{w}}}{\delta}
$$

Here $\Delta C_{\mathrm{w}}$ is radiolabeled water concentration difference within a layer of the $\mathrm{SC}$ membrane in $\mathrm{g} / \mathrm{cm}^{3}, \delta$ is SC membrane thickness, in $\mathrm{cm}$. Water partition coefficient $\left(K_{\mathrm{m}}\right)$ is defined as,

$$
K_{\mathrm{m}}=\frac{C_{\mathrm{w}}}{C_{\mathrm{ext}}} .
$$

From Eqs. (3), (4) and (5),

$$
D=\frac{k_{\mathrm{p}} \cdot \delta}{K_{\mathrm{m}}} .
$$

By assuming a constant partial molar volume for water one finds ${ }^{27}$

$$
\delta=\frac{\delta_{\mathrm{dry}}}{1-\frac{C_{\mathrm{W}}}{\rho_{\mathrm{W}}}}
$$

A dry tissue thickness $\delta_{\text {dry }}=9.55 \mu \mathrm{m}=0.000955 \mathrm{~cm}$ was employed in order to match partially hydrated $(13.4 \mu \mathrm{m})$ and fully hydrated $(43.4 \mu \mathrm{m}) \mathrm{SC}$ thicknesses calculated in Wang et al. ${ }^{33}$ for an SC membrane comprised of 15 alternating corneocyte and lipid layers.

\section{Dynamic water sorption model for isolated stratum corneum}

In this scenario dry SC tissue absorbs water from moist environment, increasing in thickness and local water concentration, $C_{w}(z, t)$. Since the absorption occurs symmetrically from both sides, we need to consider only half of the SC thickness. We choose the center of the SC to be $z$ $=0$ and the moving surface to be $z=\delta(t)$. A schematic diagram is shown in Fig. 1 . 
This is a special case of the water swelling model presented elsewhere, ${ }^{27}$ with no flux at $z=0$. Under the assumption that the water partial molar volume in the SC is equal to the pure water molar volume, the rate of change of $\delta$ is:

$$
\frac{d \delta}{d t}=\frac{f_{\delta}}{\rho_{\mathrm{w}}}
$$

where $f_{\delta}$ is the water flux at $z=\delta(t)$. The governing equation for water transport is:

$$
\frac{\partial C_{\mathrm{w}}}{\partial t}=\frac{\partial}{\partial z}\left(D \frac{\partial C_{\mathrm{w}}}{\partial z}\right)
$$

In Eq. (9), $C_{w}=C_{w}(z, t)$ is the local water concentration in $g / \mathrm{cm}^{3}$. Diffusivity varies with depth $z$ and time $t$ due to the evolving water concentration gradient within the SC. We choose to express this dependence through a function $D\left(C_{\mathrm{w}}\right)$, which is equivalent to saying that local water diffusivity, $D$, responds instantaneously to changes in local water concentration, $C_{\mathrm{w}}$, and is not otherwise dependent on distance and time. If $D$ increases exponentially with increasing $C_{\mathrm{w}}$, which is plausible at low hydration, ${ }^{4}$ then

$$
D=D_{0}+\left(D_{\max }-D_{0}\right)\left(1-e^{-\alpha C_{\mathrm{w}}}\right)
$$

$D_{0}\left(\mathrm{~cm}^{2} / \mathrm{s}\right), D_{\max }\left(\mathrm{cm}^{2} / \mathrm{s}\right)$, and $\alpha\left(\mathrm{cm}^{3} / \mathrm{g}\right)$ are parameters which will be estimated by fitting Eq. 10 to the experimental water sorption data.

The boundary conditions for a sorption experiment are:

$$
\begin{array}{ll}
\frac{\partial C_{\mathrm{w} 0}}{\partial z}=0 & \text { at } z=0 \\
f_{\delta}=D \frac{\partial C_{\mathrm{w} \delta}}{\partial z}-C_{\mathrm{w} \delta} \frac{f_{\delta}}{\rho_{\mathrm{w}}} & \text { at } \quad z=\delta(t)
\end{array}
$$

The model considers the growing SC thickness over time and changing diffusivity with respect to water content. It is implemented within the gPROMS ${ }^{\mathrm{TM}}$ software platform. This dynamic 
model can potentially yield a more accurate estimation of diffusivity than the models used previously in the skin literature, e.g. that of Liron et al., ${ }^{5}$ in which constant diffusivity and thickness are assumed for each equilibrium value of skin hydration, $C_{\mathrm{w}}^{\mathrm{eq}}$. The $\mathrm{gPROMS}^{\mathrm{TM}}$ platform facilitates this process by relieving the analyst of the burden of explicitly coding the moving boundary problem embodied in Eqs. 1-12.

\section{DATA ANALYSIS}

Existing data from the skin permeability literature were reanalyzed in terms of model equations 1-12 to obtain several independent estimates of water diffusivity in SC at different water contents. The data and their analysis are described below.

\section{SC equilibrium water content}

The primary source for the selected SC water adsorption isotherm was the analysis presented by Kasting and Barai. ${ }^{25}$ These workers collected water sorption data for human SC from six different sources and analyzed them according to four isotherm models. We selected the Guggenheim-Anderson-deBoer (GAB) model, called "modified BET" in Ref. 25. However, we chose a calorimetrically-determined value ${ }^{26}$ of the adsorption enthalpy constant $c$ (Eq. 1) rather than the value estimated by Kasting and Barai. The GAB parameters (Eq. 1) were thus $c=4.39$, $k=1 / 1.01$, and $V_{m}=0.0386 \mathrm{~g}$ water $/ \mathrm{g}$ dry tissue. This curve is shown as a solid blue line in Fig. 2. The choice of $c=4.39$ vs. $c=10.4$ as given by Kasting and Barai ${ }^{25}$ has very little impact on the selected isotherm above a relative humidity of 0.1 . El Shimi and Princen ${ }^{3}$ and Liron et al. ${ }^{5}$ also presented SC water sorption isotherms for human and porcine skin, respectively, that were not included in Kasting and Barai's analysis. These data are also shown in Fig. 2. The Liron et al. data are represented by a linear isotherm for $\mathrm{RH}<0.6$ as described in the figure caption. It may 
be seen from this comparison that the equilibrium SC water content estimated from these three sources as a function of $\mathrm{RH}$ is very similar.

\section{Steady state water permeability and diffusivity}

Blank et. al. ${ }^{2}$ conducted steady state water permeability experiments at four levels of relative humidity (RH) with human SC samples from three subjects. Johnson et. al. ${ }^{13}$ collected values of water permeability for fully hydrated SC from 8 sources. The values determined in these studies are listed in Table 1.

$<$ Insert Figure 2> and Refs. 3,5,25,26

$<$ Insert Table $1>$ and Refs. 2,13

SC water content was calculated from Eqs. 1 and 2, water partition coefficient from Eq. 5, and SC thickness from Eq. 7. Water diffusivity was then calculated from Eq. 6. The results are given in Table 2.

$<$ Insert Table 2>

\section{Dynamic water sorption analysis}

Several researchers ${ }^{3,5,6,9}$ have studied the dynamics of SC water sorption and/or desorption by recording the skin weight change over time while exposing it to water vapor. In this paper, the data of El-Shimi and Princen ${ }^{3}$ and Liron et al. ${ }^{5}$ are analyzed in detail. These data are shown in Figs. 3 and 4, respectively. The other two studies will be discussed.

In both experiments the SC surface was exposed to air with a certain constant relative humidity. Our starting assumption was that surface water concentration $\left(C_{\mathrm{w} \delta}\right)$ was instantly in equilibrium with moist air and there was no gas phase mass transfer resistance, i.e. $C_{\mathrm{w} \delta}=C_{\mathrm{w} \delta}^{\mathrm{eq}}$. However, we were not able to obtain good fits to all the experimental data under this 
assumption. In order to better match the model to the data, we considered the mass transfer resistance from bulk air to the SC surface,

$$
f_{\delta}=k_{g} \frac{\left(a_{\mathrm{w} \delta}-R H\right) p_{\mathrm{sat}}^{\circ} \mathrm{MW}}{R T}
$$

Here $k_{\mathrm{g}}=0.318 \mathrm{~cm} / \mathrm{s}$ is the mass transfer coefficient based on the model of Frasch and Bunge, ${ }^{34}$ $p_{\text {sat }}^{\circ}=4.76 \mathrm{kPa}$ is the saturated water vapor pressure at skin surface temperature, ${ }^{35} T=(32+$ 273.2) $\mathrm{K}$, and $\mathrm{MW}=18 \mathrm{~g} / \mathrm{mol}$ is water molecular weight. Recall that $a_{\mathrm{w} \delta}$ is water activity at the skin surface and $C_{\mathrm{w} \delta}$ is in equilibrium with $a_{w \delta}$ through the GAB isotherm, Eqs. 1 and 2 . This correction was particularly important for high relativity humidity data, e.g. the $\mathrm{RH}=90 \%$ and 95\% data in Fig. 3.

\section{$<$ Insert Figure $3>$ and Ref. 3 \\ $<$ Insert Figure $4>$ and Ref. 5}

For the data of Liron et al. (Fig. 4), we considered that surface water concentration relaxes exponentially from an initial value of zero to its equilibrium value, $C_{\mathrm{w} \delta}^{\mathrm{eq}}$, as calculated from the GAB isotherm,

$$
C_{\mathrm{w} \delta}=C_{\mathrm{w} \delta}^{\mathrm{eq}}\left(1-e^{-\beta t}\right)
$$

Equation 14 was also applied by Liron et al. ${ }^{5}$ The initial conditions were thus:

$$
\begin{aligned}
& C_{\mathrm{w}}=0 \\
& \delta=\delta_{\text {dry }}
\end{aligned}
$$

Liron et al. cite a dry tissue half-thickness of $8 \mu \mathrm{m}$ for their porcine skin samples, so we used this value to analyze their data. El-Shimi and Princen did not state the half-thickness for their human skin samples. We assumed a value of $7 \mu \mathrm{m}$, which is consistent with the partially 
hydrated SC thickness of 13-14 $\mu \mathrm{m}$ assumed in recent skin permeability models. ${ }^{13,33,36}$ including our own.

All of the data from each study, i.e. Fig. 3 or Fig. 4, were analyzed simultaneously using the parameter estimation capability in gPROMS, to obtain the three parameters in Eq. 10. From El Shimi's data (Fig. 3), we found $D_{0}=(0.0157 \pm 0.0016) \times 10^{-9} \mathrm{~cm}^{2} / \mathrm{s}, D_{\max }=(0.237 \pm 0.006) \times 10^{-9}$ $\mathrm{cm}^{2} / \mathrm{s}, \alpha=(68 \pm 66) \mathrm{cm}^{3} / \mathrm{g}$, and for Liron et al., $D_{0}=(0.043 \pm 0.014) \times 10^{-9} \mathrm{~cm}^{2} / \mathrm{s}, D_{\max }=(0.50 \pm$ $0.15) \times 10^{-9} \mathrm{~cm}^{2} / \mathrm{s}, \alpha=(2.93 \pm 0.35) \mathrm{cm}^{3} / \mathrm{g}$. The uncertainties given here are standard parameter errors estimated by the gPROMS optimization routine. The fitted curves are shown in Figs. 3 and 4.

The analysis for the El Shimi data is valid for $C_{\mathrm{w}} \leq 0.4 \mathrm{~g} / \mathrm{cm}^{3}$ and that for Liron et al. for $C_{\mathrm{w}} \leq$ $0.1 \mathrm{~g} / \mathrm{cm}^{3}$. Neither of these datasets approach the value $C_{\mathrm{w}}=0.78 \mathrm{~g} / \mathrm{cm}^{3}$ estimated for fully hydrated skin (Line 5 in Table 2). The use of Eq. 10 with the fitted parameters for these datasets furthermore leads to lower values of $D$ than estimated for the steady-state water permeability data in Table 2. Consequently, we added a second exponential term to Eq. 10 to allow the value of $D$ to increase rapidly at high $C_{\mathrm{w}}$ and match the fully hydrated skin value of 1.9 $\times 10^{-9} \mathrm{~cm}^{2} / \mathrm{s}$. Consideration of both the transient datasets (Figs. 3 and 4 ) in combination with the steady state data (Table 2) yielded the following phenomenological equation for water diffusivity in human SC (Model 1):

$$
D \times 10^{9}=0.4331-0.3765 e^{-9.6215 C_{w}}+0.00006428 e^{12.873 C_{w}}
$$

where $D$ is in $\mathrm{cm}^{2} / \mathrm{s}$, and $C_{\mathrm{w}}$ is water content in $\mathrm{g} / \mathrm{cm}^{3}$. The steady state data and the developed models are shown in Figure 5. The wide variation in the experimental data, even among the three human skin donors studied by Blank et al., ${ }^{2}$ reflects the uncertainty associated with this 
analysis. There are no data for $0.4<C_{\mathrm{w}}<0.78 \mathrm{~g} / \mathrm{cm}^{3}$, and Model 1 is purely empirical. However, we show later that it is consistent with a mechanistic model for water diffusivity in a biphasic SC structure (Model 2, Appendix 1) and also a much earlier analysis of transepidermal water loss ${ }^{8}$ (Model 3, Appendix 2) for $0.2 \leq C_{w} \leq 0.78 \mathrm{~g} / \mathrm{cm}^{3}$, which covers the common range of hydration for human skin in vivo. The Model 2 result is shown as the dashed red curve in Fig. 5. $<$ Insert Figure 5> and Refs. 3,5

We considered also the water sorption and desorption data from human SC obtained by Anderson et al. ${ }^{9}$ and that on porcine SC obtained by Rauma et al. ${ }^{6}$ These data were not easily reconciled with the results shown in Fig. 5; the former gave much lower values of $D$, the latter much higher values. Anderson et al. ${ }^{9}$ performed their study in a Cahn electrobalance. We postulate that the kinetics in this very still environment were dominated by the gas phase mass transfer resistance (cf. Eq. 13) rather than by the SC. Rauma et al. ${ }^{6}$ used a novel thermogravimetric method to measure sorption/desorption kinetics for water and several volatile organic solvents. Baseline relative humidity was low (45.5\%) in these studies due to the mixing of dry and wet airstreams; the corresponding SC water sorption volume calculated from Eq. 1 is $V=0.055 \mathrm{~g}\left(\mathrm{H}_{2} \mathrm{O}\right) / \mathrm{g}$ (dry tissue). Water uptake and loss was rapid, but was limited to $4 \%$ of the SC baseline weight. Based on a GAB isotherm prediction of $V=0.380 \mathrm{~g}\left(\mathrm{H}_{2} \mathrm{O}\right) / \mathrm{g}(\mathrm{dry}$ tissue) at $91 \% \mathrm{RH}$, an equilibrium uptake of $31 \%$ [calculated as $100 \% \times(1.380-1.055) / 1.055$ ] would have been expected for human SC obeying Eq. 1. Consequently the water uptake in the Rauma et al. study was much lower than the isotherm prediction. We have no firm explanation for this difference other than to note that equilibrium water uptake in the flow calorimetry study of Yadav et al. ${ }^{26}$ was also much lower than that predicted by Eq. 1 . It is possible that the 
maximum $\mathrm{RH}$ values achieved in both the Rauma and Yadav studies were less than the target values. For $\mathrm{RH}$ values $>90 \%$ the sorption curve is very steep, so a small error in estimated $\mathrm{RH}$ leads to a large difference in water content (cf. Fig. 2).

\section{DISCUSSION}

Despite the large body of literature describing the impact of water on the skin and measurement thereof, ${ }^{37}$ quantitative analyses of water transport in skin are relatively rare. Most have considered either steady-state transport in the context of Fick's First Law ${ }^{2,8,11}$ or dynamic transport in the context of the diffusion equation (Fick's Second Law), ${ }^{3,5,6}$ with or without a variable diffusion coefficient. Stockdale's imaginative 1978 analysis, ${ }^{8}$ although based on external water concentrations rather than internal values, yielded a water diffusivity function having much in common with Eq. 17. We show in Appendix 2 that Stockdale's analysis leads to a water diffusivity profile very similar to Eq. 17 for $0.2 \leq C_{\mathrm{w}} \leq 0.78 \mathrm{~g} / \mathrm{cm}^{3}$ if the external concentrations are replaced by internal water concentrations calculated from Eqs. 1 and 2.

An alternative approach was taken by vanLogtestijn et al. ${ }^{12}$ These workers considered three regions of the SC with varying degrees of diffusive resistance, and obtained relationships for diffusive resistance to water transport as a function of depth in the SC. Water concentrations were taken from confocal Raman measurements rather than being estimated from in vitro isotherm data. The results were combined in a dynamic model capable of predicting the time course of water concentrations in skin following product application.

Lacking in all the cited analyses is a convective term that accounts for swelling and deswelling of the SC as its hydration state changes. This is a large correction, not a minor one, since the tissue absorbs up to three times its weight in water at full hydration. ${ }^{25}$ Consequently, 
models that do not account for swelling are destined to fail quantitatively when used to describe the dynamics of water transport in the SC. We recently developed a dynamic model for SC water transport with the capability of properly accounting for swelling. ${ }^{27}$ To minimize complexity we considered the SC to be homogeneous rather than building in a complex layer structure as in vanLogetestijn et al. ${ }^{12}$ The model was designed for compatibility with the microscopic model of Wang et al. ${ }^{33,38}$ at the two hydration states focused on by these authors.

The water diffusivity versus concentration profile referred to herein as Model 1 (Eq. 17 and Fig. 5) is the second key component of the dynamic swelling model, the first being proper accounting for convection. Increased swelling leads to increased water diffusivity, a feature common to all of the models discussed. It is interesting and somewhat reassuring that Model 1, the microscopic model (Model 2, Eqs. A1-A7) and a re-parameterized version of Stockdale's ${ }^{8}$ model (Model 3, Eq. A8) all yield similar water diffusivity profiles for $C_{\mathrm{w}}>0.2 \mathrm{~g} / \mathrm{cm}^{3}$. The common principle in these approaches is that diffusivity is directly related to the degree of swelling. In terms of Model 2, this result is rationalized as follows: (1) Corneocytes swell as the water content of the SC increases, extending the keratin fiber matrix within each cell. The local diffusivity, $D_{\text {cor, }}$ increases in a manner consistent with water diffusion in other keratin matrices (Fig. A1 and Eq. A6). The shape of this curve can be qualitatively explained on the basis of either hindered diffusion or free volume theories. ${ }^{4,39}$ (2) As the corneocytes swell, the lipids are strained. The strain is not present in the multilaminate model presented here (Appendix 1), but it is evident from two or three-dimensional brick-and-mortar models that it must occur. ${ }^{13,33,38,40}$ The strain disorders the lipid packing in the bilayers, resulting in an increase in permeability to water and other solutes. According to Model 2, water diffusivity increases approximately 
seven-fold as the tissue swells from the partially hydrated $\left(C_{\mathrm{w}}=0.30 \mathrm{~g} / \mathrm{cm}^{3}\right)$ to the fully hydrated $\left(C_{\mathrm{w}}=0.78 \mathrm{~g} / \mathrm{cm}^{3}\right)$ state.

An examination of Fig. 5 (cf. also Tables 1 and 2) shows that the database on which the water diffusivity models discussed herein are built is sparse and highly variable. Much of the variability is related to inter-subject differences in skin permeability. For example, the Blank et al. $^{2}$ study shows more than a two-fold variation in skin permeability to water for three human skin donors, whereas the eight full hydration studies summarized by Johnson et al. ${ }^{13}$ show an even larger variation. The human skin water permeability values reported by Johnson et al. span the range $1.4 \times 10^{-4} \mathrm{~cm} / \mathrm{h}$ to $1.71 \times 10^{-3} \mathrm{~cm} / \mathrm{h}$, a variation of more than 12 -fold. ${ }^{13}$ These studies and those of Blank et al. were conducted using standard methodology for steady-state experiments. So it is not possible to make a precise calculation of water diffusivity in human SC; one must instead search for a "central tendency". We believe Model 1 (Eq. 17) captures this tendency based on available water diffusion data. The individual terms contributing to Eq. 17 should not be over analyzed; rather, the shape of the curve should be examined. It is reassuring that Models 2 and 3 give similar shapes in the physiologically relevant range of skin hydration, $0.20 \leq C_{\mathrm{w}} \leq 0.78 \mathrm{~g} / \mathrm{cm}^{3}$, and that independent analyses of skin permeability to other compounds also show steep rises as water activity at the SC surface approaches that at the base of the SC. ${ }^{20,31,32}$ The concept that this rise is driven by strain and resulting disruption of the SC lipids is compelling. ${ }^{20}$

While the premises and mathematical framework are different, our recommended Model 1 is not inconsistent with the picture presented by vanLogetestijn et al. ${ }^{12}$ Given the differences seen in equilibrium swelling of different layers of the $\mathrm{SC}^{14,19}$ and the recognized loss of lipids 
from the outer SC during desquamation, ${ }^{41}$ it is likely that transverse anisotropy of the SC is a feature that must eventually be included in a detailed model. The present framework, supported by suitable evidence, could accommodate such an addition.

\section{CONCLUSIONS}

Water diffusivity in human stratum corneum (SC) is a strong function of water content and increases rapidly as the tissue swells when both sides are exposed to high water activity (or relative humidity). Three different approaches to describing this dependence yield similar diffusivity values for water concentrations in the range $0.2 \leq C_{\mathrm{w}} \leq 0.78 \mathrm{~g} / \mathrm{cm}^{3}$. Agreement between approaches is poor for $C_{\mathrm{w}}<0.2 \mathrm{~g} / \mathrm{cm}^{3}$; however, this SC hydration range is not frequently encountered in vivo. The nonlinear diffusivity function given by Eq. 17 (Model 1) explains the nonlinear water concentration versus depth profiles measured instrumentally and leads to the prediction that dehydration rates are more rapid than hydration rates, as discussed elsewhere. ${ }^{27}$

\section{ACKNOWLEDGEMENTS}

$\mathrm{XL}$ acknowledges a post-doctoral fellowship from the Procter \& Gamble Company. GBK acknowledges support from the Cefic Long Range Initiatives program (LRI B13), the NSF GOALI program (Award No. 1335822), and prior support from the NIOSH extramural research program (Award No. R01 OH007529), which enabled the development of these concepts. The views expressed here are those of the authors and have not been endorsed by the external sponsors of this work. 


\section{Appendix 1: Microscopic model for water diffusivity in the stratum corneum (Model 2)}

We take as the starting point a two-dimensional brick-and-mortar model of the SC as shown in Fig. A1, Panels (a) and (b). It is well recognized that this geometry degenerates into a much simpler $A B A B$ multilaminate membrane with the $A$ layers representing $S C$ corneocytes and the B layers intercellular lipids (Panel (c)), if diffusion through the lipid "necks" separating the corneocytes can be neglected. ${ }^{4,38,42,43}$ Wang et al. ${ }^{38}$ show that this limit applies for permeants having dimensionless diffusion parameters $R$ and $\sigma$ such that $R>100$ and $\sigma<1.5$. According to the parameterization developed by Wang et al., these limits are easily met for water diffusing through either partially hydrated $\left(C_{\mathrm{w}}=0.37 \mathrm{~g} / \mathrm{cm}^{3}\right)$ or fully hydrated $\left(C_{\mathrm{w}}=0.78 \mathrm{~g} / \mathrm{cm}^{3}\right)$ skin. These values correspond to average water weight percentages in the SC of $30 \%$ and $74 \%$, respectively. We estimate it will continue to hold for SC hydration values as low as $C_{\mathrm{w}}=0.2$ $\mathrm{g} / \mathrm{cm}^{3}\left(16 \mathrm{wt} \% \mathrm{H}_{2} \mathrm{O}\right)$, which is as dry as the outer SC ever gets in vivo. Consequently, the multilaminate model is a widely applicable simplifying assumption.

$<$ Insert Figure A1> and Refs. 13,33,38

In this picture, the steady state permeability, $P_{\mathrm{sc}}$, and diffusive resistance, $R_{\mathrm{sc}}$, of the composite membrane with respect to a pure water phase are given by

$P_{\mathrm{sc}}=\frac{1}{R_{\mathrm{sc}}}=\frac{D_{\mathrm{sc}} K_{\mathrm{sc} / \mathrm{w}}}{\delta}$

$R_{\mathrm{sc}}=R_{\text {lip }}+R_{\text {cor }}=\frac{\delta_{\text {lip }}}{D_{\text {lip }} K_{\text {lip } / \mathrm{w}}}+\frac{\delta_{\text {cor }}}{D_{\text {cor } K_{\text {cor } / \mathrm{w}}}}$

Development of the model entails estimating the microscopic parameters for the lipid and corneocyte phases to obtain $R_{\mathrm{sc}}$ and $\mathrm{P}_{\mathrm{sc}}$, calculating $K_{\mathrm{sc} / \mathrm{w}}$ as the weighted sum of $K_{\text {lip } / \mathrm{w}}$ and $K_{\text {cor } / \mathrm{w}}$, $K_{\mathrm{sc} / \mathrm{w}}=\left(K_{\text {lip } / \mathrm{w}} \delta_{\text {lip }}+K_{\text {cor } / \mathrm{w}} \delta_{\text {cor }}\right) / \delta$. 
Note that $K_{\mathrm{sc} / \mathrm{w}}$ is simply equal to $C_{\mathrm{w}}$ (Eq. 2 ), as it is calculated with respect to pure water. Total thickness $\delta$ is calculated from Eq. 7. The effective diffusion coefficient $D_{\text {sc }}$ can then be calculated by rearranging Eq. A1.

The model membrane as a whole swells with water uptake according to Eq. 7. All of the swelling is assumed to occur in the corneocyte phase; lipid sublayer thickness and the partition coefficient for water between the lipids and the external phase do not change with hydration. Based on the model of Wang et al., ${ }^{33}$ the total lipid thickness is $\delta_{\text {iip }}=15 \times 0.091 \mu \mathrm{m}=1.37 \mu \mathrm{m}$ and the water partition coefficient in this phase is $K_{\text {lip } / w}=0.0328$. The total corneocyte thickness is then

$$
\delta_{\text {cor }}=\delta-\delta_{\text {lip }}
$$

and their partition coefficient with respect to water is (from Eq. A3)

$K_{\text {cor } / \mathrm{w}}=\left(K_{\mathrm{sc} / \mathrm{w}} \delta-K_{\text {lip } / \mathrm{w}} \delta_{\text {lip }}\right) / \delta_{\text {cor }}$

It remains to estimate values for $D_{\text {lip }}$ and $D_{\text {cor. }}$. Both values are expected to increase with increasing hydration of the tissue, for reasons discussed elsewhere. ${ }^{4,38}$ We chose to estimate $D_{\text {cor }}$ from water diffusivities measured in a variety of keratin substrates as discussed by Kasting et al. ${ }^{4}$ The data are shown in Fig. A2, plotted as a function of water volume fraction in the tissue, $\phi_{1}$.

\section{$<$ Insert Figure A2>}

Rather than constructing a theoretical model for this behavior (see Ref. 4), we fit an empirical, sigmoidal curve to these data in order to accurately represent them. In doing this we omitted several $D_{0}$ values at higher water content from Watt's study of wool as shown in Fig. A2, and we heavily weighted the single value of $D_{0}$ at $\phi_{1}=0.806$ representing fully hydrated 
skin. This was done to reflect the extensive amount of data leading to this value (Line 5 of Table 2). The equation resulting from this procedure was

$$
\log D_{0}\left(\mathrm{~cm}^{2} / \mathrm{s}\right)=-19.28+14.59 /\left(1+\exp \left[\frac{-\left(\phi_{1}+0.0730\right)}{0.1563}\right]\right)
$$

As a final step, we associated the values of $\phi_{1}$ with $K_{\text {cor/w }}$ and $D_{0}$ with $D_{\text {cor }}$ (cf. Eq. A2).

The value of $D_{\text {lip }}$ was determined from the steady state diffusivity data in Table 2 and Fig. 5 according to the following procedure. A value, $D_{\text {lip }}^{\min }$, and corresponding SC thickness, $\delta_{\min }$, was chosen in order to match the effective $D$ values (i.e. $D_{\text {sc }}$ ) and thickness for the low hydration data in Table 2. Similarly, values of $D_{\text {lip }}^{\max }$ and $\delta_{\max }$ were chosen to match the fully hydrated $D$ value in Table 2. The value of $D_{\text {sc }}$ was calculated from Eqs. A1-A6. We made the assertions that (1) $D_{\text {lip }}$ increases linearly with increasing strain on the SC lipids, and that (2) the strain is proportional to the amount of swelling relative to SC with a water content equal to the driest samples in Table $2\left(C_{\mathrm{w}}=0.067 \mathrm{~g} / \mathrm{cm}^{3}\right)$. The value of $D_{\text {lip }}$ was thus calculated as

$$
D_{\text {lip }}=D_{\text {lip }}^{\min }+\frac{\left(\delta-\delta_{\min }\right)}{\left(\delta_{\max }-\delta_{\min }\right)}\left(D_{\text {lip }}^{\max }-D_{\text {lip }}^{\min }\right)
$$

The selected parameter values were $\delta_{\min }=10.2 \mu \mathrm{m}, D_{\mathrm{lip}}^{\min }=2.02 \times 10^{-10} \mathrm{~cm}^{2} / \mathrm{s}$ and $\delta_{\max }=43.4$ $\mu \mathrm{m}, D_{\text {lip }}^{\max }=1.40 \times 10^{-9} \mathrm{~cm}^{2} / \mathrm{s}$. Transverse water diffusivity in the SC lipids was thereby found to increase by a factor of $1.40 \times 10^{-9} / 2.02 \times 10^{-10}=6.9$ as the tissue swells from the partially hydrated to fully hydrated state. The final microscopic water diffusivity model calculated from Eqs. A1-A7 is shown as Model 2in Fig. A3.

$<$ Insert Figure $A 3>$ and Ref. 8 


\section{Appendix 2: Heuristic analysis for water diffusivity in the stratum corneum (Model 3)}

Stockdale in $1978^{8}$ was already familiar with the work of El Shimi and Princen ${ }^{3}$ and also the then-ongoing debate regarding the impact of an occlusive film on transepidermal water loss (TEWL). ${ }^{23,24}$ He collected TEWL data as a function of relative humidity (RH) from a number of sources and analyzed them according to what might be called a heuristic (or intuitive) model for water transport in the SC. His central finding was that the collective data could be described by an equation of the form

$$
\frac{D}{D_{0}}=0.175\left(\frac{C_{0}}{C_{0}-C}\right)+0.46
$$

where $D_{0}$ and $C_{0}$ were water diffusivities and concentrations at a standard state, taken to be RH $=100 \%$ at the inner surface of the SC and $\mathrm{RH}=0 \%$ at the outer surface. The water concentrations considered in Stockdale's analysis were derived from external water vapor concentrations in a manner that cannot be defended.

Nevertheless, the functional form of Eq. A8 predicts a steep rise in water diffusivity $D$ as water concentration approaches a limiting value, $C_{0}$. In order to test Eq. 8 in a manner consistent with current thinking, we took $C_{0}$ to be the water concentration predicted from the GAB isotherm (Eqs. 1 and 2) at $a_{\mathrm{w}}=1$, and $D_{0}$ to be the diffusivity value leading to agreement of Eq. A8 with the fully hydrated skin diffusivity value in Table 2 at $a_{\mathrm{w}}=0.996$. The results were $C_{0}$ $=0.835 \mathrm{~g} / \mathrm{cm}^{3}$ and $D_{0}=0.60 \times 10^{-9} \mathrm{~cm}^{2} / \mathrm{s}$. The resulting water diffusivity profile is shown as Model 3 in Fig. A2. 


\section{Figure captions}

Figure 1. Diagram of dynamic water sorption model (symmetrical swelling from both sides of tissue). There is no flux of water across the plane of $z=0$ and an inward-directed flux $f_{\delta}$ across the outer boundary of the SC, $z=\delta(t)$.

Figure 2. Water sorption isotherms in human and porcine SC. The blue line is the GAB model (Eq. 1) combining parameter values from Kasting and Barai ${ }^{25}$ and Yadav et al. ${ }^{26}$ as described in the text. Filled circles $(\bullet)$ represent equilibrium human skin data from El Shimi and Princen. ${ }^{3}$ The dashed line represents the porcine skin data from Liron et al. ${ }^{5}$ The equation for the line is $v$ $=0.19 \times \mathrm{RH}(\mathrm{RH}<0.6)$.

Figure 3. Water sorption kinetics in human abdominal SC(adapted from El Shimi and Princen ${ }^{3}$ ). The dashed curves represent the data and the solid curves show the model fit.

Figure 4. Water sorption kinetics in porcine SC (adapted from Liron et al. ${ }^{5}$ ). The dashed curves represent the data and the solid curves show the model fit.

Figure 5. Water diffusivity vs. water content in stratum corneum. The plotted data are taken from Table 2. The purple and black dashed curves represent fits of Eq. 10 to the data in El Shimi and Princen ${ }^{3}$ and Liron et al. ${ }^{5}$, respectively. The green line shows the phenomenological model (Model 1) calculated according to Eq. 17. The dashed red line is the microscopic model (Model 2) given by Eqs. A1-A7. Model 2 is only valid when diffusion through the corneocytes overwhelms that through the lipid necks separating corneocytes. This region corresponds approximately to $C_{\mathrm{w}}>0.2$. The dotted red line shows the extrapolation of Model 2 into the region of uncertainty at low values of $C_{w}$, as discussed in Appendix 1.

Figure A1. (a) Two-dimensional brick-and-mortar model of the stratum corneum (after Johnson et al. ${ }^{13}$ (b) Detail of intercellular lipid structure in the trapezoidal brick-and-mortar model of

Wang et al. ${ }^{33,38}$ The diagram shows their Model 2 lipid arrangement. (c) An ABAB multilaminate membrane is the limit of either Johnson et al. or Wang et al. if diffusion through the corneocytes is much greater than that through the lipid "necks" separating the corneocytes within each layer. All models depicted assume $\mathrm{N}=15$ layers of each coexisting phase.

Figure A2. Water diffusivity, $D_{0}$, plotted as a function of water volume fraction, $\phi_{1}$, in a variety of keratin matrices. ${ }^{4}$ The red line shows the sigmoidal model calculated according to Eq. A6. The open circles represent water diffusivities in wool that were excluded from the fit.

Figure A3. Water diffusivity in the stratum corneum as a function of its water content according to the three models discussed in the text. The solid blue line (Model 1 ) is the phenomenological model in the main text (Eq. 17 and Fig. 2). The dashed green line (Model 2) represents the microscopic model calculated according to Eqs. A1-A7. The dotted red line (Model 3 ) is derived from Stockdale's ${ }^{8}$ heuristic analysis as discussed in Appendix 2. 


\section{References}

1. Blank IH 1952. Factors which influence the water content of the stratum corneum. J Invest Dermatol 18:433-440.

2. Blank IH, Moloney J, Emslie AG, Simon I, Apt C 1984. The diffusion of water across the stratum corneum as a function of its water content. J Invest Dermatol 82:183-194.

3. El-Shimi AF, Princen HM 1978. Water vapor sorption and desorption behavior of some keratins. Colloid Polym Sci 256(3):209-217.

4. Kasting GB, Barai ND, Wang T-F, Nitsche JM 2003. Mobility of water in human stratum corneum. J Pharm Sci 92(11):2326-2340.

5. Liron Z, Clewell HJ, McDougal JN 1994. Kinetics of water vapor sorption in porcine stratum corneum. J Pharm Sci 83(5):692-698.

6. Rauma M, Isaksson TS, Johanson $\mathrm{G}$ 2006. A new technique to assess dermal absorption of volatile chemicals in vitro by thermal gravimetric analysis. Toxicol in Vitro 20:1183-1189.

7. Roskos KV, Guy RH 1989. Assessment of skin barrier function using transepidermal water loss: effect of age. Pharmaceut Res 6(11):949-953.

8. Stockdale M 1978. Water diffusion coefficients versus water activity in stratum corneum: a correlation and its implications. J Soc Cosmet Chem 29:625-639.

9. Anderson RL, Cassidy JM, Hansen JR, Yellin W 1973. The effect of in vivo occlusion on human stratum corneum hydration-dehydration in vitro. J Invest Dermatol 61(6):375-379.

10. Anderson RL, Cassidy JM, Hansen JR, Yellin W 1973. Hydration of stratum corneum. Biopolymers 12:2789-2802.

11. Pirot F, Berardesca E, Kalia YN, Singh M, Maibach HI, Guy RH 1998. Stratum corneum thickness and apparent water diffusivity: facile and noninvasive quantitation in vivo. Pharmaceut Res 15(3):492494.

12. van Logtestijn MDA, Domínguez-Hüttinger1 E, Stamatas GN, Tanaka1 RJ 2015. Resistance to water diffusion in the stratum corneum Is depth-dependent. PLoS ONE 10(2):e0117292.

13. Johnson ME, Blankschtein D, Langer R 1997. Evaluation of solute permeation through the stratum corneum: lateral bilayer diffusion as the primary transport mechanism. J Pharm Sci 86(10):11621172.

14. Bouwstra JA, de Graaff A, Gooris GS, Nijsse J, Wiechers JW, van Aelst AC 2003. Water distribution and related morphology in human stratum corneum at different hydration levels. J Invest Dermatol 120:750-758.

15. Bouwstra JA, Gooris GS, van der Spek JA, Bras W 1991. Structural investigations of human stratum corneum by small angle X-ray scattering. J Invest Dermatol 97:1005-1012. 
16. Caspers P, Lucassen G, Bruining H, Puppels G 2000. Automated depth-scanning confocal Raman microspectrometer for rapid in vivo determination of water concentration profiles in human skin $\mathrm{J}$ Raman Spectrosc 31:813-818.

17. Caspers PJ, Lucassen GW, Carter EA, Bruining HA, Puppels GJ 2001. In vivo confocal Raman microspectroscopy of the skin: noninvasive determination of molecular concentration profiles. J Invest Dermatol 116(3):434-442.

18. Warner RR, Boissy YL, Lilly N, Spears MJ, McKillop KL, Stone KJ 1997. Water disrupts stratum corneum lipid lamellae. J Invest Dermatol 108(4):644.

19. Warner RR, Stone KJ, Boissy YL 2003. Hydration disrupts human stratum corneum ultrastructure. J Invest Dermatol 120(2):275-284.

20. Bjorklund S, Engblom J, Thuresson K, Sparr E 2010. A water gradient can be used to regulate drug transport across skin. J Control Rel 143(2):191-200.

21. Grice K, Sattar H, Baker H 1972. The effect of ambient humidity on transepidermal water loss. J Invest Dermatol 58:343-346.

22. Scheuplein RJ, Ross LW 1974. Mechanism of percutaneous absorption V. Percutaneous absorption of solvent deposited solids. J Invest Dermatol 62(4):353-360.

23. Cooper ER, Van Duzee BF 1976. Diffusion theory analysis of transepidermal water loss through occlusive films. J Soc Cosmet Chem 76(27):555-558.

24. Weil I, Princen HM 1977. Diffusion theory analysis of transepidermal water loss through occlusive films. J Soc Cosmet Chem 28:481-484.

25. Kasting GB, Barai ND 2003. Equilibrium water sorption in human stratum corneum. J Pharm Sci 92(8):1624-1631.

26. Yadav S, Thiel SW, Kasting GB, Pinto NG 2009. Thermodynamics of water interactions with human stratum corneum. II. Interpretation via the Guggenheim-Anderson-deBoer isotherm. Chem Eng Sci 64:1480-1487.

27. Li X, Johnson R, Weinstein B, Wilder E, Smith E, Kasting GB 2015. Dynamics of water transport and swelling in human stratum corneum. Chem Eng Sci, 138:164-172.

28. Ostrenga J, Steinmetz C, Poulson B, Yett S 1971. Significance of vehicle composition II: Prediction of optimal vehicle composition. J Pharm Sci 60:1180-1183.

29. Berner B, Juang R-H, Mazzenga GC 1989. Ethanol and water sorption into stratum corneum and model systems. J Pharm Sci 78(6):472-476.

30. Berner B, Mazzenga GC, Otte JH, Steffens RJ, Juang R-H, Ebert CD 1989. Ethanol:water mutually enhanced transdermal therapeutic system II: skin permeation of ethanol and nitroglycerin. J Pharm Sci 78(5):402-407. 
31. Bunge AL, Persichetti JM, Payan JP 2012. Explaining skin permeation of 2-butoxyethanol from neat and aqueous solutions. Int J Pharm 435(1):50-62.

32. Frasch HF, Barbero AM, Dotson GS, Bunge AL 2014. Dermal permeation of 2-hydroxypropyl acrylate, a model water-miscible compound: effects of concentration, thermodynamic activity and skin hydration. Int J Pharm 460:240-247.

33. Wang T-F, Kasting GB, Nitsche JM 2006. A multiphase microscopic diffusion model for stratum corneum permeability. I. Formulation, solution and illustrative results for representative compounds. J Pharm Sci 95(3):620-648.

34. Frasch HF, Bunge AL 2015. The transient dermal exposure II: post-exposure absorption and evaporation of volatile compounds. J Pharm Sci 104: 1499-1507.

35. Weast RC editor 1984. Handbook of Chemistry and Physics. 65th ed., Boca Raton, FL: CRC Press.

36. Chen L, Lian G, Han L 2010. Modeling transdermal permeation. Part I. Predicting skin permeability of both hydrophobic and hydrophilic solutes. AIChE J 56(5):1136-1146.

37. Fluhr JW, Elsner P, Berardesca E, Maibach HI. 2005. Bioengineering of the skin: Water and the stratum corneum. CRC Press.

38. Wang T-F, Kasting GB, Nitsche JM 2007. A multiphase microscopic model for stratum corneum permeability. II. Estimation of physicochemical parameters and application to a large permeability database. J Pharm Sci 96(11):3024-3051.

39. Gunt HB, Miller MA, Kasting GB 2007. Water diffusivity in human nail plate. J Pharm Sci 96(12):3352-3362.

40. Naegel A, Heisig M, Wittum G 2009. A comparison of two- and three-dimensional models for the simulation of the permeability of human stratum corneum. Eur J Pharm Biopharm 72(2):332-338.

41. Kasting GB, Miller MA 2006. Kinetics of finite dose absorption through skin 2. Volatile compounds. J Pharm Sci 95(2):268-280.

42. Berner B, Cooper ER 1983. Asymptotic solution for nonsteady-state diffusion through oil-water multilaminates. J Membrane Sci 14:139-145.

43. Berner B, Cooper ER 1984. Desorption out of an oil-water multilaminate membrane. J Control Rel 1(2):149-152. 
Figure 1

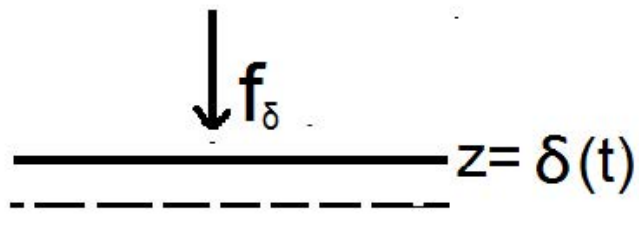

$-\cdot \cdot-\cdot-z=0$

$-\cdots-C z=0$ 
Figure 2

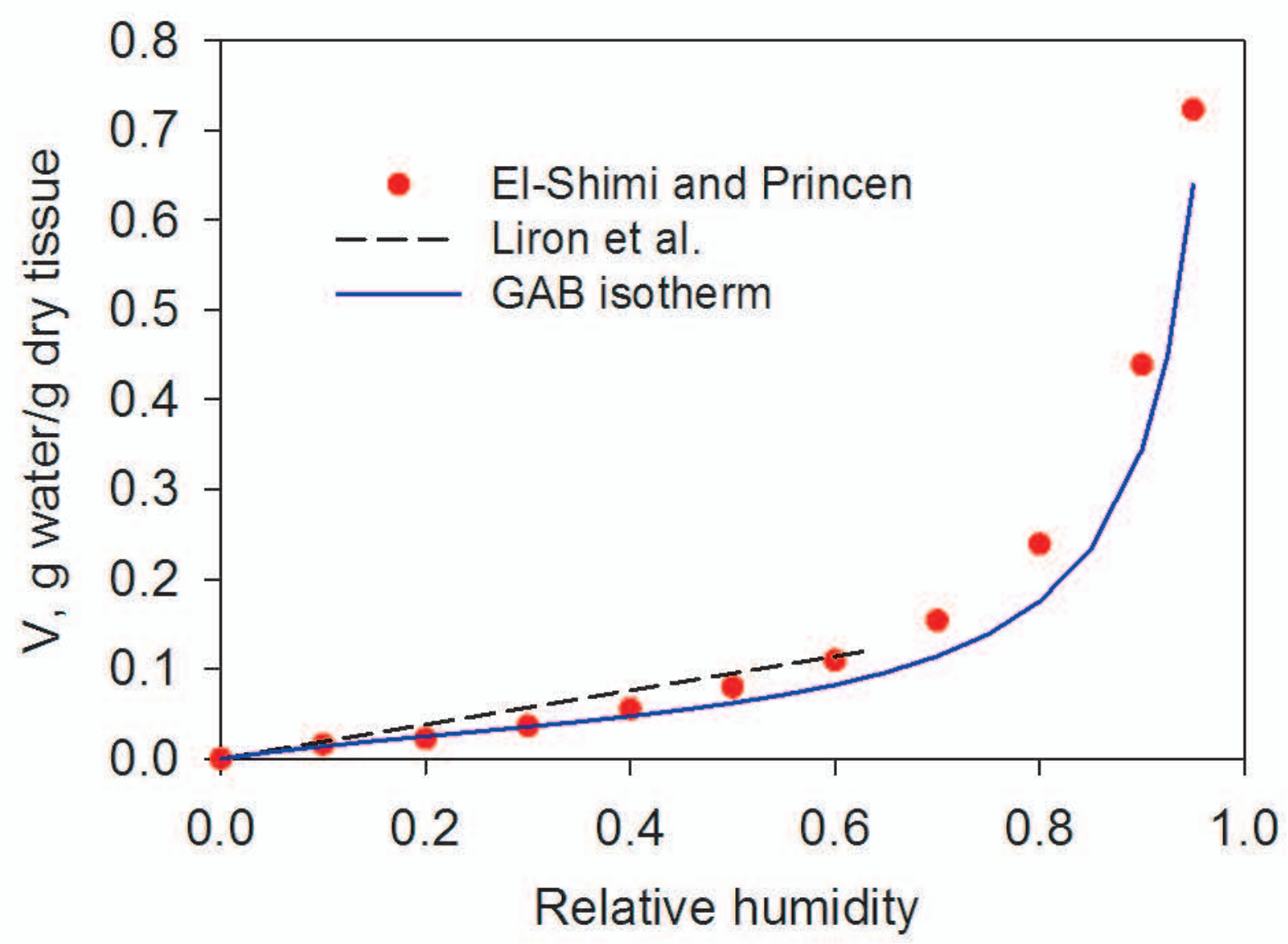


Figure 3

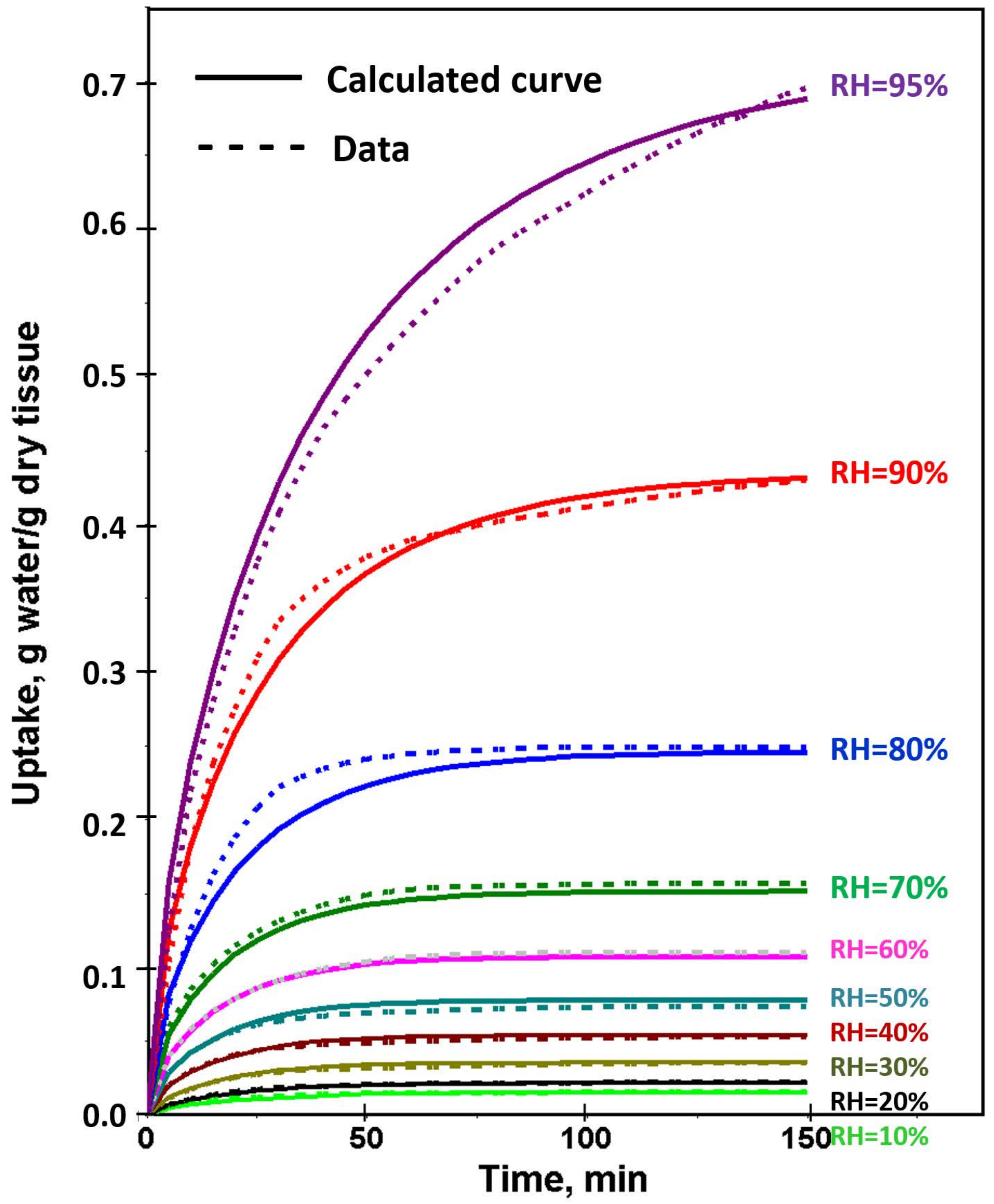


Figure 4

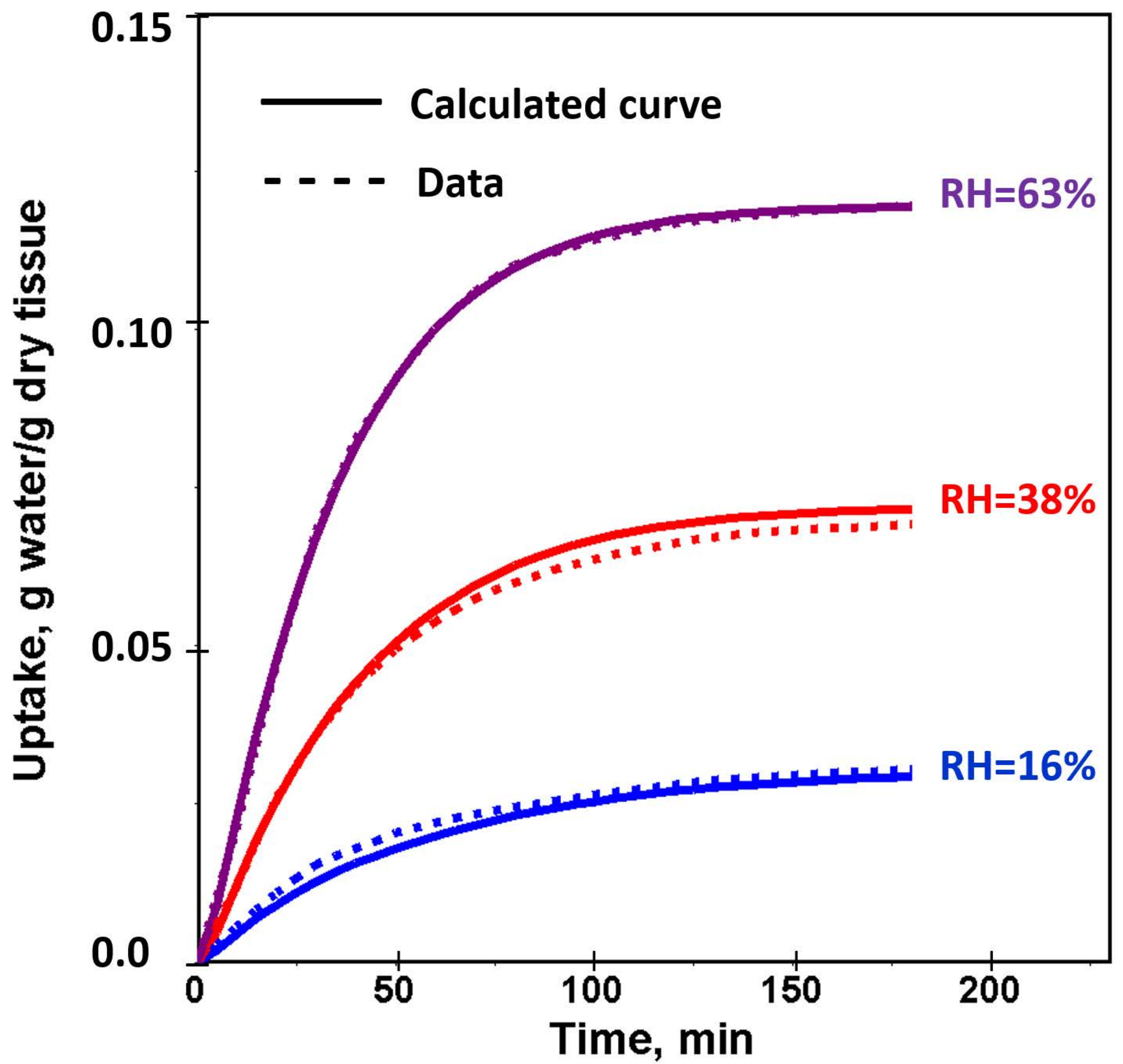




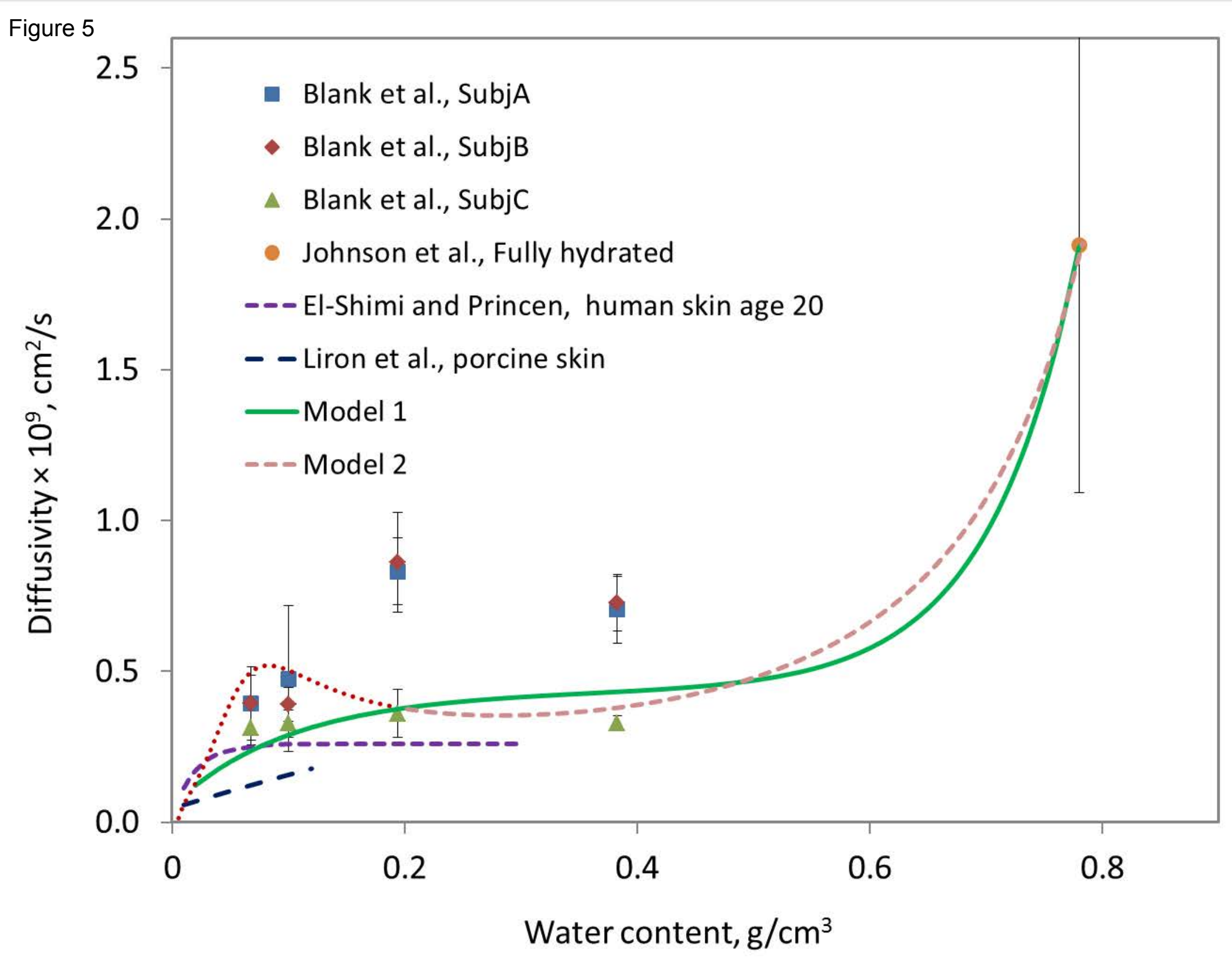


Table 1. Steady state water permeability values $\left(k_{\mathrm{p}}\right)$

\begin{tabular}{|c|c|l|l|l|c|}
\hline \multirow{2}{*}{$\begin{array}{c}\mathrm{RH} \\
\%\end{array}$} & $\mathrm{C}_{\mathrm{ext}}$, & \multicolumn{3}{|c|}{$k_{\mathrm{p}} \times 10^{7}, \mathrm{~cm} / \mathrm{s}$} & Ref. \\
\cline { 3 - 5 } $\mathrm{g} / \mathrm{cm}^{3}$ & Subj A & Subj B & Subj C & \multirow{2}{*}{ (Tables I and II) } \\
\hline 46 & 0.71 & $0.39 \pm 0.12$ & $0.39 \pm 0.09$ & $0.31 \pm 0.06$ & \\
\hline 62 & 0.78 & $0.61 \pm 0.31$ & $0.50 \pm 0.07$ & $0.42 \pm 0.06$ & \\
\hline 81 & 0.87 & $1.66 \pm 0.22$ & $1.72 \pm 0.33$ & $0.72 \pm 0.16$ & \\
\hline 93 & 0.94 & $1.97 \pm 0.31$ & $2.03 \pm 0.26$ & $0.92 \pm 0.07$ & \multirow{2}{*}{3.4 (Table 1) } \\
\hline 99.6 & 1 & \multicolumn{3}{|c|}{$3.4 \pm 1.5^{\mathrm{a}}$} \\
\hline
\end{tabular}

${ }^{a}$ Mean \pm SD of eight studies. 
Table 2. Water diffusivities estimated from permeability data in Table 1.

\begin{tabular}{|c|c|c|c|c|c|c|}
\hline \multirow[t]{2}{*}{$\begin{array}{l}\mathrm{RH} \\
\%\end{array}$} & \multirow[t]{2}{*}{$\begin{array}{c}C_{\mathrm{w}}, \mathrm{g} / \mathrm{cm}^{3} \\
\text { Eq.(2) }\end{array}$} & \multirow[t]{2}{*}{$\begin{array}{c}K_{\mathrm{m}} \\
\text { Eq. }(5)\end{array}$} & \multirow[t]{2}{*}{$\begin{array}{l}\delta, \mu \mathrm{m} \\
\text { Eq.(7) }\end{array}$} & \multicolumn{3}{|c|}{$\begin{array}{c}D \times 10^{9}, \mathrm{~cm}^{2} / \mathrm{s} \\
\text { Eq. }(6)\end{array}$} \\
\hline & & & & Subj $A$ & Subj B & Subj $C$ \\
\hline 46 & 0.068 & 0.096 & 10.2 & $0.42 \pm 0.13$ & $0.42 \pm 0.10$ & $0.33 \pm 0.06$ \\
\hline 62 & 0.102 & 0.13 & 10.6 & $0.50 \pm 0.26$ & $0.41 \pm 0.06$ & $0.35 \pm 0.05$ \\
\hline 81 & 0.194 & 0.22 & 11.8 & $0.88 \pm 0.12$ & $0.91 \pm 0.18$ & $0.38 \pm 0.09$ \\
\hline 93 & 0.383 & 0.41 & 15.5 & $0.75 \pm 0.12$ & $0.77 \pm 0.10$ & $0.35 \pm 0.03$ \\
\hline 99.6 & 0.78 & 0.78 & 43.4 & \multicolumn{3}{|c|}{$1.9 \pm 0.8$} \\
\hline
\end{tabular}

